\title{
Planetary Interior in the Laboratory
}

R. Chau, M. Bastea, A.C. Mitchell, R.W. Minich, W.J. Nelis

January 31, 2003

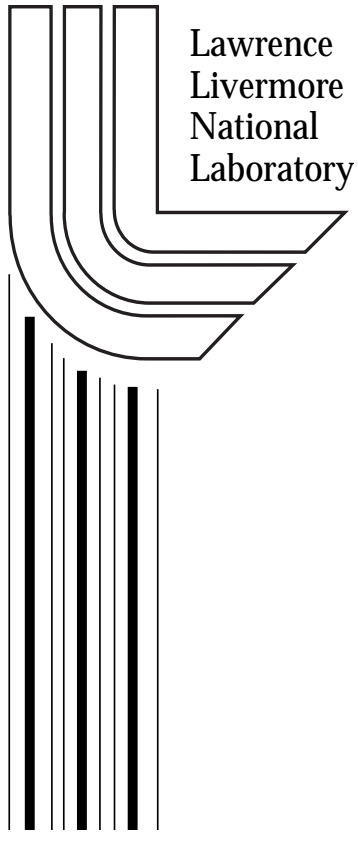




\section{DISCLAIMER}

This document was prepared as an account of work sponsored by an agency of the United States Government. Neither the United States Government nor the University of California nor any of their employees, makes any warranty, express or implied, or assumes any legal liability or responsibility for the accuracy, completeness, or usefulness of any information, apparatus, product, or process disclosed, or represents that its use would not infringe privately owned rights. Reference herein to any specific commercial product, process, or service by trade name, trademark, manufacturer, or otherwise, does not necessarily constitute or imply its endorsement, recommendation, or favoring by the United States Government or the University of California. The views and opinions of authors expressed herein do not necessarily state or reflect those of the United States Government or the University of California, and shall not be used for advertising or product endorsement purposes.

This work was performed under the auspices of the U. S. Department of Energy by the University of California, Lawrence Livermore National Laboratory under Contract No. W-7405-Eng-48.

This report has been reproduced directly from the best available copy.

Available electronically at http://www.doe.gov/bridge

Available for a processing fee to U.S. Department of Energy and its contractors in paper from

U.S. Department of Energy

Office of Scientific and Technical Information

P.O. Box 62

Oak Ridge, TN 37831-0062

Telephone: (865) 576-8401

Facsimile: (865) 576-5728

E-mail: reports@adonis.osti.gov

Available for the sale to the public from

U.S. Department of Commerce

National Technical Information Service 5285 Port Royal Road

Springfield, VA 22161

Telephone: (800) 553-6847

Facsimile: (703) 605-6900

E-mail: orders@ntis.fedworld.gov

Online ordering: http://www.ntis.gov/ordering.htm

OR

Lawrence Livermore National Laboratory

Technical Information Department's Digital Library

http://www.llnl.gov/tid/Library.html 


\section{Planetary Interiors in the Laboratory}

R. Chau, M. Bastea, A. C. Mitchell, R. W. Minich, and W. J. Nellis

Lawrence Livermore National Laboratory,

University of California, Livermore, CA 94550 


\section{INTRODUCTION}

Understanding the interiors of the giant planets Uranus and Neptune is an important aspect of planetary physics. The planets Neptune and Uranus accreted from the planetary ices, water $\left(\mathrm{H}_{2} \mathrm{O}\right)$, methane $\left(\mathrm{CH}_{4}\right)$ and, ammonia $\left(\mathrm{NH}_{3}\right)$, in solar proportions $\left(56 \% \mathrm{H}_{2} \mathrm{O}\right.$, $\left.36 \% \mathrm{CH}_{4}, 8 \% \mathrm{NH}_{3}\right)$. Neptune and Uranus are thought to have a three-layer structure: a small rocky core around which there is a thick layer of ice comprising two thirds of the planetary mass and an outer atmosphere of molecular hydrogen and helium [1]. The top of the ice layer is estimated to be at $30 \mathrm{GPa}$ and $2500 \mathrm{~K}$ while the base of the ice layer is at $600 \mathrm{GPa}$ and $7000 \mathrm{~K}$. At these conditions, the ice in the central regions of Uranus and Neptune is a warm dense fluid, which is highly degenerate. While the planetary fluids invoke many fascinating condensed matter issues, two open and fundamental questions about the interiors of Uranus and Neptune are the origin of their large and complex magnetic fields and their mass distributions [2].

Information on Neptune and Uranus have come from measurements aboard the Voyager II spacecraft and ground based observations. Unfortunately, the majority of measurements, except gravitational and magnetic measurements, do not give information about the interiors of these planets. Models of the interiors of Uranus and Neptune rely on matching measured gravitational moments using existing equation of state (EOS) data of the planetary fluids. Within the error bars of the gravitational moments, a number of models of the interiors of Uranus and Neptune have been proposed that fit the gravitational moments. However, these models produce somewhat different mass distributions [3, 4]. In fact, two layer and three layer models of Uranus and Neptune are both acceptable within the error bars. The interpretations of the interiors of Uranus and Neptune are not constrained because information about planetary fluids at high pressures and temperatures is incomplete. Moreover, effects such as dissociation into ions and Insulator-Metal transitions are not included in existing EOS data for the planetary constituents. Determining the state of matter (ionic, metallic, etc.) via conductivity measurements will dictate the choice of EOS in modeling planetary interiors.

The goal of this project is to measure the electrical transport properties of the planetary fluids $\left(\mathrm{H}_{2} \mathrm{O}, \mathrm{NH}_{3}\right.$, and $\left.\mathrm{CH}_{4}\right)$ at conditions representative of the deep interior of Uranus and Neptune. Because these fluids dissociate at high temperatures and pressures, it is equally 
important to understand their basic constituents $\mathrm{H}, \mathrm{O}, \mathrm{N}$, and $\mathrm{C}$. The electrical transport properties will allow direct interpretation of the large complex magnetic fields on Uranus and Neptune. Also, the electrical conductivity is sensitive to dissociation into ions and transitions from insulating and semiconducting and to metallic states and thus gives information on the state of matter. Theoretical calculations of the electrical conductivity along the planetary isentrope of Uranus predict ionic conduction in water and ammonia and metallization above $300 \mathrm{GPa}[5]$.

Conditions within the interiors of Neptune and Uranus can be recreated in Earth based laboratories using dynamic compression (gas guns, laser-driven shocks, and explosive drivers) and, possibly, static compression. Gas gun drivers using a reverberating shock wave can produce quasi-isentropic compression i.e. substantially higher pressures and densities at lower temperatures than on the Hugoniot. The quasi-isentropic compression technique has been demonstrated in the metallization of hydrogen. We will use the reverberating shock wave technique to achieve quasi-isentropic compression, which yields states comparable to those in the deep interior of Neptune and Uranus.

\section{SCOPE OF WORK}

We measured electrical conductivities, pressures, and temperatures of the planetary fluids and their basic constituents up to $200 \mathrm{GPa}$ and $10000 \mathrm{~K}$ along planetary isentropes achieved with a reverberating shock wave generated by a gas gun. The electrical conductivities of these planetary fluids will give us insight into their local structure of the fluid via its conduction mechanism and, secondly, the value of the conductivity will give insight into how the planetary magnetic fields are generated. The first phase of this project was divided into two parallel goals. The first goal was to measure the electrical conductivities of the primary planetary fluids, $\mathrm{H}_{2} \mathrm{O}, \mathrm{NH}_{3}$, and $\mathrm{CH}_{4}$. The second goal was to understand the electrical conductivity of the individual components, $\mathrm{N}_{2}$ and $\mathrm{O}_{2}$ with $\mathrm{H}_{2}$ already measured, since we expect fluids such as $\mathrm{H}_{2} \mathrm{O}$ and $\mathrm{CH}_{4}$ to dissociate under high pressures and temperatures. The second phase of this project was to then study a mixture representative of the planetary interior of Neptune and Uranus.

To generate the high pressures and temperatures within a planet in the laboratory, we used the $28 \mathrm{~m}$ two-stage light gas gun at LLNL. To reach higher pressures and densities than 


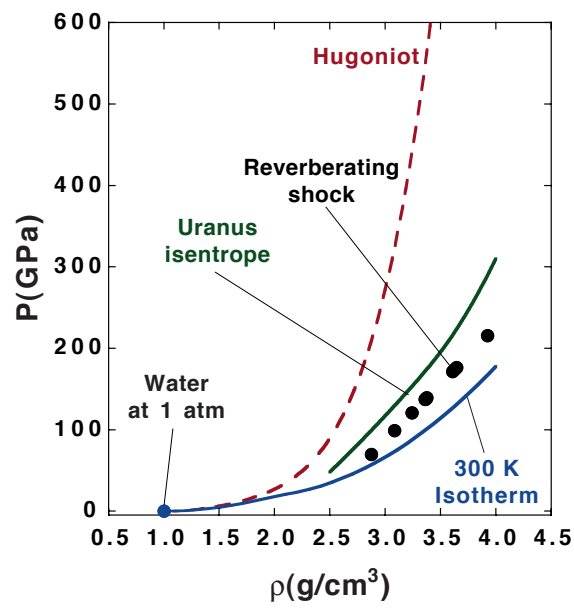

FIG. 1: A comparison of the pressure-density states achieved in our reverberation experiments versus single shock and the planetary isentrope of Uranus

in earlier works, we used a reverberating shock wave technique $[6,7]$. In our reverberating shock experiments, the liquid sample is sandwiched between two insulating stiff sapphire anvils. A metal projectile was propelled to velocities as high as $6.8 \mathrm{~km} / \mathrm{s}$ using a two-stage light-gas-gun. The impact generates a strong shock wave in the first anvil. This strong shock wave creates a weaker initial shock in the liquid sample that reverberates in the sample between the two stiff anvils. The first shock in the water is strong and compresses the water from ambient pressure to about $60 \%$ of the final pressure and accounts for twothirds the final temperature. The subsequent shocks are relatively weak shocks and compress the sample typically another factor of two. The pressure rings up to the inital pressure in the first sapphire anvil. This step-wise compression produced by the reverberating shock wave is quasi-isentropic. This reverberating technique achieves densities and temperatures much closer to the planetary isentropes of Neptune and Uranus.

The electrical conductivities on all of the experiments were made using either a constant current ot constant voltage circuit, depending on the resistance of the sample. The targets were designed to give a 200-250 ns time window to measure the resistance of the sample. This time was sufficient for the reverberations to reach a final pressure and perform the measurement but short enough before effects such as instabilities or diffusion to affect the results. The inertial confinement of the sample allows both high pressures and temperatures 


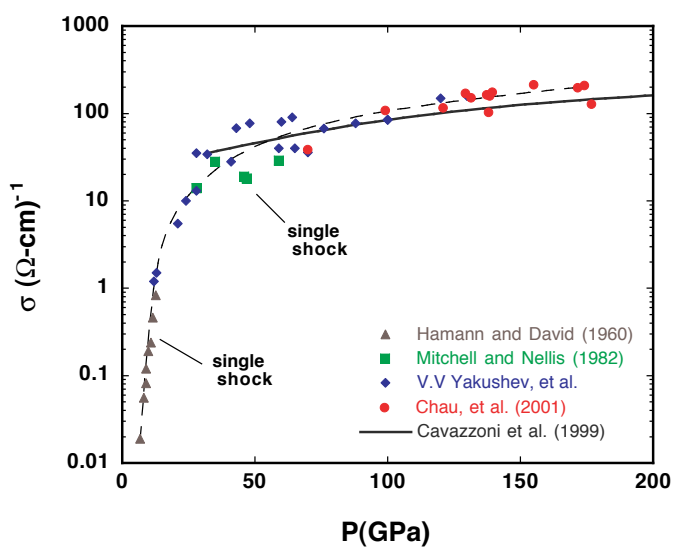

FIG. 2: The electrical conductivity of water plotted as functions of shock pressure.

to be maintained simultaneously.

\section{TECHNICAL ACHIEVEMENTS}

The first material we studied was water. Water is the primary component of the ice found within Uranus and Neptune and is the most important material for understanding the planetary interior. The conductivity data for $\mathrm{H}_{2} \mathrm{O}$ are plotted vs shock pressure in Fig. 2 as well as previous single shock data at lower pressures [8-11]. The electrical conductivity at lower pressures ( $\mathrm{P} \leq 60 \mathrm{GPa})$ increases rapidly with increasing pressures because of chemical ionization. Between 70 and $180 \mathrm{GPa}$, the electrical conductivity increases slowly from 30 to $210(\Omega-\mathrm{cm})^{-1}$. The conductivity of $\mathrm{H}_{2} \mathrm{O}$ up to $180 \mathrm{GPa}$ is consistent with a purely ionic conduction mechanism. A small contribution from electronic conduction is possible but cannot be determined from our measurements. Our measurments are consistent with the $\mathrm{H}_{2} \mathrm{O}$ dissociating into $\mathrm{H}^{+}$and $\mathrm{OH}^{-}$. The measured values of the electrical conductivity of water is larger than the estimated value of $10(\Omega-\mathrm{cm})^{-1}$ needed to sustain a dynamo and hence a self sustaining planetary magnetic field.

Since water was shown to dissociate, it became important understand the electrical conductivity of the elements that comprise water. The electrical conductivity of oxygen is necessary to interpret the electrical conductivity of $\mathrm{H}_{2} \mathrm{O}$. The electrical conductivity data 


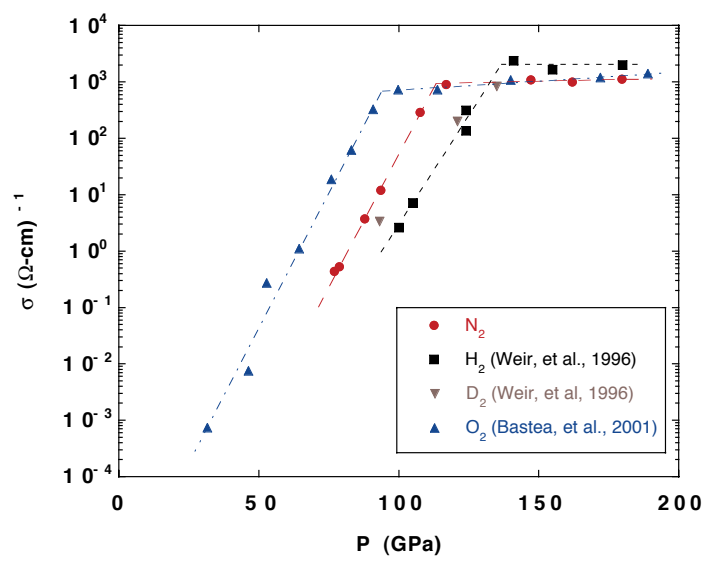

FIG. 3: The electrical conductivity of $\mathrm{H}, \mathrm{N}$, and $\mathrm{O}$ plotted as functions of shock pressure.

for oxygen is shown in fig.3 The conductivity of oxygen is similar to that of hydrogen. Below 100 GPa, oxygen exhibits a semiconducting behavior with a thermally activated mobility gap. Above $100 \mathrm{GPa}$, oxygen becomes metallic and reaches the minimum metallic conductivity. The nonmetal-metal transition in oxygen occurs at a much lower pressure than for hydrogen. However, the transition to a metallic state occurs at a pressure comparable to that required to metallize oxygen under static pressures at ambient temperature [14]. We found that the conductivity was strongly temperature dependent. At a given pressure, the electrical conductivity measured in our shock reverberation experiments was much lower than that observed in previous single shock experiments.

At the pressures found within the deep interiors of Uranus and Neptune, water is not simply a mixture of hydrogen and oxygen. The conductivity of water up to $200 \mathrm{GPa}$ is purely ionic while oxygen and hydrogen are electronic. Molecular dynamics simulation performed at LLNL and elsewhere show that the state of the fluid is dynamic in nature. Molecules breakup and recombine on a fast time scale (tens of picoseconds) with many transient species possible. The fluid that comprise the planetary interior is likely very complex due to the large number of atomic species. One of the implications is that the behavior of the planetary interior, which is a mixture, may be dominated by chemical processes.

Nitrogen is one of the basic constituents of ammonia. It is also a diatomic gas like hydrogen and oxygen and is expected to undergo a non-metal to metal transition like hy- 
drogen and oxygen. The nitrogen triple bond is very strong and nitrogen is expected to have a metallization pressure higher than oxygen and perhaps even hydrogen. The electrical conductivity of nitrogen is shown in figure 3 to $180 \mathrm{GPa}$. The electrical conductivity of nitrogen is very similar to those of hydrogen and oxygen. From $80 \mathrm{GPa}$ to $120 \mathrm{GPa}$, nitrogen is semiconducting and can be described by the same exponential form used to describe the semiconducting state in fluid hydrogen [7]. The electrical conductivity of nitrogen reaches minimum metallic conductivity at $\sim 120 \mathrm{GPa}$. The conductivity above the transition, 1000 $(\Omega-\mathrm{cm})^{-1}$, is esentially pressure and temperature independent. The electrical conductivity of hydrogen [6] and oxygen [19] is shown in comparison to that of nitrogen in figure 3. Except for slightly different transition pressures to the metallic fluid, the electrical conductivity of all three are essentially the same. The slight differences in the conductivity in the semiconducting region is attributed to the different temperature history and mobility gaps of each material.

The analysis of the nitrogen data required the development and implementation of a new EOS for nitrogen. The existing EOS do not take into account the observed dissociative transition in nitrogen. This phase transition was treated using a Mie-Gruniesen EOS with a negative Gruniesen gamma in the vicinity of the phase transition. It is likely that hydrogen will behave in a similar manner at higher pressures. The existence of a negative gamma has large implications on the thermal transport properties within planets.

We have been able to explain the non-metal to metal transition in $\mathrm{N}_{2}, \mathrm{O}_{2}$, and $\mathrm{H}_{2}$ to first order using ideas of Mott. The Mott criterion argues that a transition to a metallic state will occur when the ratio of the mean distance between nearest neighbors and the effective Bohr radius of the molecule or atoms is 0.25 . Shown in figure 4 is the electrical conductivity of $\mathrm{N}_{2}, \mathrm{O}_{2}$, and $\mathrm{H}_{2}$ plotted versus the Mott scaling parameter $\mathrm{a}^{*} \mathrm{D}_{m}^{-1 / 3}$, where $\mathrm{a}^{*}$ is the effective Bohr radius and $\mathrm{D}_{m}$ is the nearest neighbor distance. All three fluids metallize with a Mott parameter between 0.25 and 0.3 . This result suggests that the metallization of these fluids is chiefly due to band overlap of valence electrons on adjacent particles caused by the high compression. We found that the metallization of expanded fluid Rb and Cs to behave in and identical manner to the highly compressed fluid, H, N, and O. This suggests that the metallization of simple atomic fluids under planetary interior conditions has a common mechanism.

We found that the scaling of the metallization densities in $\mathrm{H}, \mathrm{N}$, and $\mathrm{O}$ can be explained 


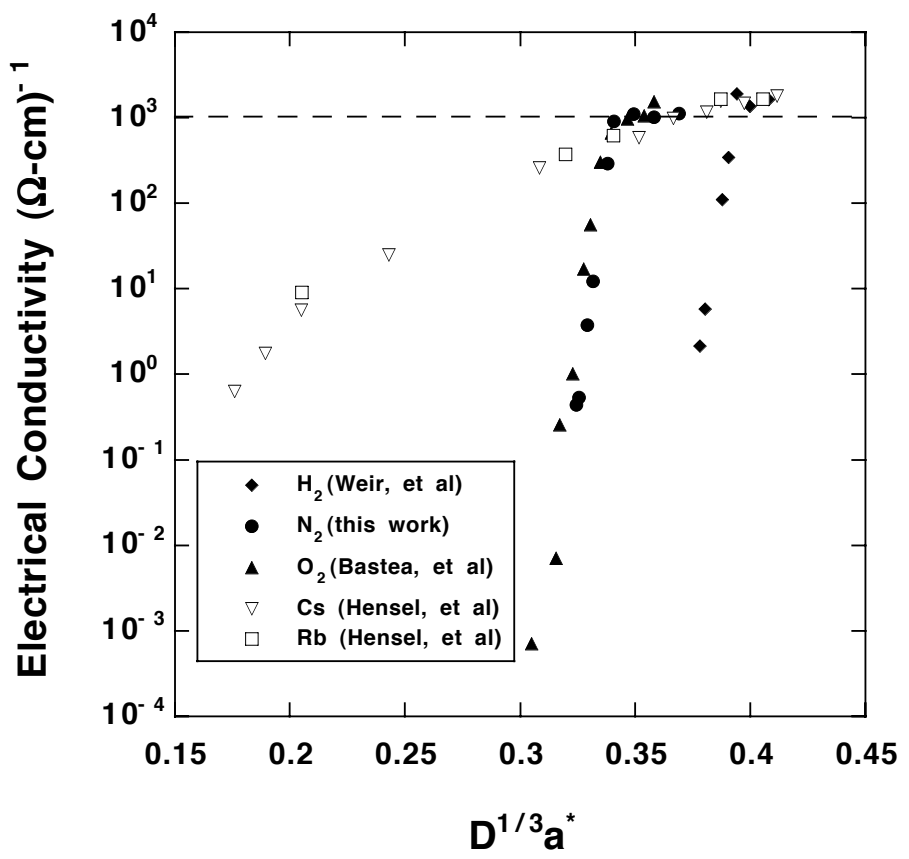

FIG. 4: The electrical conductivity of $\mathrm{H}, \mathrm{N}, \mathrm{O}, \mathrm{Rb}$, and $\mathrm{Cs}$ plotted as functions of scaled density.

in terms of the radial charge desnity distributions. In essence, the larger atoms have a greater extent of the radial charge densities, thus less pressure is required to obtain sufficient overlap of the charge densities of adjacent atoms for the electrons to delocalize and hence metallize. At the pressures and temperatures within plannetary interiors, nitrogen, oygen, and hydrogen are all fully dissociated and the fluid is monoatomic rather than diatomic. This seems to be an essential feature for metallization. Within the scope of this simple model, we expect that other monoatomic fluids at similar conditions such as $\mathrm{CO}, \mathrm{F}_{2}$, and others will undergo an identical metallization process barring any additional chemical interactions. 
In the case of water, which is composed of oxygen and hydrogen, the chemical interactions between different atomic species appear to prevent this metallization process.

Methane is second in abundance next to water. Methane has long been predicted to dissociate into hydrogen and carbon in the form of diamond [12, 15]. Static high pressure experiments have shown indications of dissociation [16]. The dissociated carbon is predicted to cluster and perhaps sink towards the rocky core of Neptune and Uranus. This would change the composition of the ice layer towards a more hydrogen rich composition. Previous measurements of hydrocarbons in gas gun experiments support the idea of dissociation [17]. The dissociated carbon is predicted to cluster and perhaps sink towards the rocky core of Neptune and Uranus and make the interior compositions of Uranus and Neptune more hydrogen rich. We performe the electrical conductivity measurements on liquid methane. We observed above $120 \mathrm{GPa}$ a plateau in the conductivity which corresponds presumably to the conductivity of methane itself. After tens of nanoseconds, we found the conductivity to increase monotonically in time. This behavior is consistent with the methane dissociating into hydrogen and carbon. The expected volume of carbon is probably too small to form a percolating path for conduction but the hydrogen is more conductive at those pressures and temperatures. Fig. 5 shows the voltage trace for methane shock reverberated to $127 \mathrm{GPa}$ and shows the time dependence of the electrical conductivity of methane. At the very highest pressure, we cannot observe the dissocation process. It is likely at the highest pressures, that we have overdriven the chemical dissociation. The kinetics and timescale for chemical dissociation is highly dependent of the temperature and pressure. The surprising result is that the dissociation process requires time to develop, in some cases up to $100 \mathrm{~ns}$.

The final experiment we performed for this project was to study a suitable mixture. A mixture of different fluids such as found in Uranus and Neptune is expected to have more complicated chemical processes due to the larger number of atomic species present in the mixture. For this project, we chose a mixture we refer to as "synthetic Uranus" which is a mixture of water, ammonia, and isopropyl alcohol. This mixture is mixed to have approximately the same atomic ratios of $\mathrm{H}, \mathrm{N}, \mathrm{O}$, and $\mathrm{C}$ atoms as within Uranus. The measurements have been performed but not fully analyzed at the time of this writing. The preliminary results show that the electrical conductivity has the same pressure dependence as water although the conductivity is about $20 \%$ lower than that of water. This is not surprising since water comprises up to $2 / 3$ the "synthetic Uranus" mixture. 


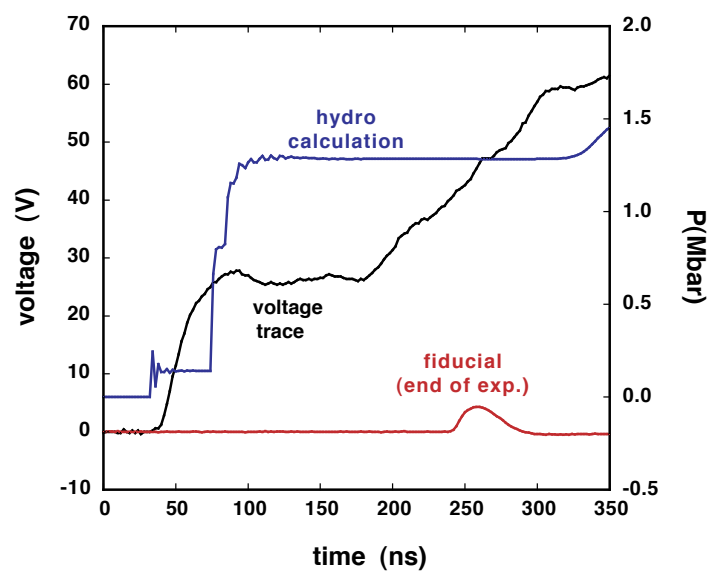

FIG. 5: A plot of sample voltage versus time for methane shocked reverberated to 127 GPa. The blue trace is a hydrocode simulation of the pressure versus time of the methane.

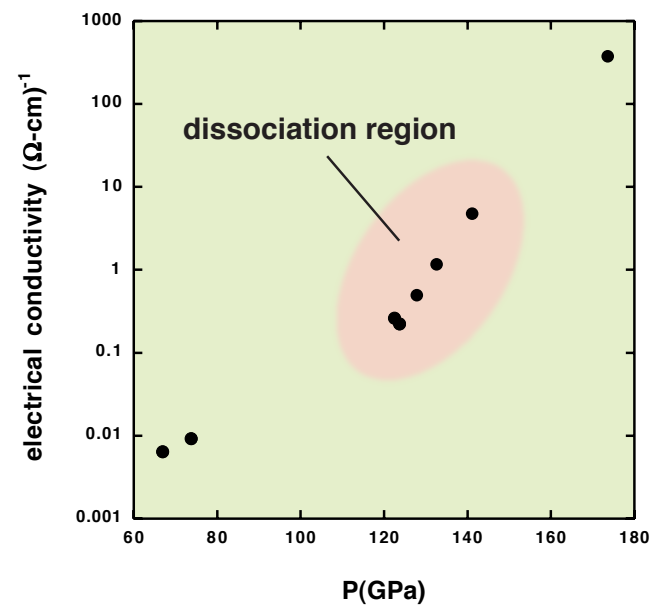

FIG. 6: Electrical conductivity versus final pressure for methane.

\section{SUMMARY}

In the three years of this project, we have provided a complete database of the electrical conductivity of planetary materials to $180 \mathrm{GPa}$. The electrical conductivities of these planetary materials now provide a basis for future modelling of planets taking into account full 
magnetohydrodynamics. By using a full magnetohydrodynamics simulation, the magnetic fields of the planets can then be taken into account. Moreover, the electrical conductivities of the planetary materials have given us insight into the structure and nature of these dense fluids. We showed that simple monoatomic fluids such as hydrogen, nitrogen, and oxygen at planetary interior conditions undergo a common metalliation process which can be explained on a simple basis of their radial charge density distributions. This model also shows that the metallization process is actually rather common and likely to take place in a number of materials such as carbon monoxide which is also present within planetary objects. On the otherhand, we have also showed that a simple two component fluid like water and methane take on much different behaviors than say nitrogen due to the chemical interactions within these systems. The dynamics of an even more complex system, "synthetic Uranus" are still being analyzed but suggest that on some levels the behavior is very simple, i.e. the electrical conductivity is essentially the same as water, but the local dynamics are very complex. This project has shed much light on the nature of electrical transport within planetary interiors but also has shown that understanding chemical processes in the complex fluids within planetary interiors to be very important. Understanding those local interactions and processes is required to gain further insight into planetart interiors.

[1] W.B. Hubbard, Science 275, 1279 (1997).

[2] D. J. Stevenson, J. of Phys., Cond. Matt. 10, 11227 (1998).

[3] W. B. Hubbard and M. S. Marley., Icarus 78, 102 (1989).

[4] M. S. Marley, P. Gomez, M. Podolak, Journal of Geophysical Research 100, 23349 (1995).

[5] C. Cavazzoni, G. L. Chiarotti, S. Scandolo, E. Tosatti, M. Bernasconi, M. Parrinello, Science 283, 44 (1999).

[6] S. T. Weir, A. C. Mitchell, and W. J. Nellis, Phys. Rev. Lett. 76, 1860 (1996).

[7] W. J. Nellis, S. T. Weir, and A. C MitchellPhys. Rev. B 59, 3434-3449 (1999).

[8] S. D. Hamann and M. Linton, Trans. Faraday Soc. 65, 2186 (1969).

[9] S. D. Hamann and M Linton, Trans. Faraday Soc. 62, 2234-2241(1966).

[10] A. C. Mitchell and W. J. Nellis J. Chem. Phys. 76, 6273-6281(1982).

[11] V. V. Yakushev, in High Pressure Research (eds Wentzcovitch, R.M., Hemley, R.J., Nellis, 
W.J., Yu, P.Y.) 121-126 (Materials Research Society, Boston, 1997).

[12] M. Ross, textitNature 292, 435 (1981).

[13] F. Ancilotto, G. L. Chiarotti, S. Scandolo, E. Tosatti, Science 275, 1288 (1997).

[14] K. Shimizu, et. al., Nature 393, 767 (1998).

[15] F. H. Ree, J. Chem. Phys. 70, 974 (1979).

[16] L. R. Benedetti, Science 286, 100 (1999).

$[17]$

[18] R. Chau, A. C. Mitchell, R. W. Minich, W. J. Nellis, J. Chem Phys. 114, 1361 (2001).

[19] M. Bastea, A. C. Mitchell, and W. J. Nellis, Phys. Rev. Lett. 86, 3108 (2001). 